\title{
A propósito de los trastornos límite
}

RESUMEN: A partir de una reflexión clínica de los trastornos límite se introduce un faro de orientación que permite dilucidar, en su seno, la neurosis y la psicosis.

PALABRAS CLAVE: Límite, neurosis, psicosis, sentido, sin sentido.
SUMMARY: Starting with a clinical reflection on the limit disorders, a guidance headlight is introduced in order to clear up, in itself, the neurosis and the psychosis.

KEY WORDS: Limit, neurosis, psychosis, sense, senseless.

Hace más de treinta años que me preocupan diversas cuestiones relacionadas con los trastornos límite. Sin embargo, mi impresión sobre este asunto es que el trastorno límite sigue gozando de una cierta imprecisión en el diagnóstico y tratamiento.

La intención de este trabajo es, por una parte, ayudar a comprender esta clínica desde la perspectiva psicoanalítica en la que trabajo; pero también indicar lo que podemos aprender de estos sujetos que viven bajo la influencia de una vivencia límite, constituida por una sensación de vacío o hendidura subjetiva que ilumina circunstancialmente su errancia en la vida. Porque también podríamos definir a estos pacientes límite como sujetos coyunturalmente errantes en la vida. Y nótese cómo resuenan en esta última frase todas esas preguntas: ¿quién soy?, ¿qué soy?, ¿qué destino me acoge y espera?, que enmarcan la vida de un sujeto y que se captan siempre incesantemente en el encuentro con este tipo de pacientes. Por otro lado digo «coyunturalmente errantes por la vida» porque, de hecho, algunos de estos pacientes, según la literatura y mi propia experiencia, pueden incluso ir mejorando espontáneamente en el trascurso de la vida. Es como si, en cierto modo, la vida y su enseñanza, que anuncia que «no todo es posible», pudiera ir dulcificando este tipo de malestar en algunos de estos pacientes que vamos a ir definiendo.

Ahora bien, si aludo a cierta dulcificación sintomática en algunos de estos sujetos, es porque en mi experiencia clínica lo que se define como trastorno límite no es para nada un grupo homogéneo, como ya veremos. Es éste un aspecto que los clínicos debemos tener muy en cuenta a la hora del encuentro con este tipo de pacientes.

No obstante hay un punto de sumo interés en estos casos raros de la clínica. Porque lo más relevante de este trastorno es que, en el desencadenamiento del cuadro y como si de una infección aguda se tratara, algunos de estos sujetos pueden quedar verdaderamente afectados por las consecuencias del revés acontecido en la búsqueda de respuestas ante lo imposible de la vida (contacto con drogas, trastornos alimentarios potencialmente graves, claudicación escolar o deterioro en su 
inserción en lo social). De ahí la importancia de un diagnóstico y tratamiento precoz para evitar, en la medida de lo posible, el rigor de todas las secuelas que puedan surgir en esa búsqueda de respuestas ante la vida.

Sin embargo, es preciso interrogar en esta nosología lo que bajo el término límite nos alumbra en estos casos, siempre complicados en la clínica, porque, a mi modo de ver, la vivencia límite de estos pacientes -que hay que entender como el encuentro traumático con lo real, esto es, con lo imposible de decir, que es también lo insoportable- y su errancia circunstancial en la vida tienen un punto de sumo interés para todos nosotros. De ahí la necesidad de poner palabras a ese encuentro del paciente con lo imposible, para así poder limitar esa vivencia traumática. Y éste es uno de los cometidos del tratamiento psicoanalítico.

Pero además la vivencia límite en estos sujetos nos permite captar siempre un vacío o agujero, que desencadena angustia, y que facilita, en ocasiones, la irrupción de un acto -la impulsividad-como modo de calmar ese vacío o de atemperar ese agujero sin nombre. Ya veremos la diferencia que planteo entre vacío y agujero en función de los diferentes tipos de pacientes que empañan el denominado trastorno límite.

Ahora bien, ¿a qué se refiere este límite? Dicho de otra manera, ¿por qué aparece el término límite para etiquetar este tipo de sufrimiento? (Y digo sufrimiento porque no hay que olvidar que la clínica es siempre lo imposible de soportar, pues tiene siempre una dimensión trágica tanto para el paciente que la vive como para el profesional que la soporta, como también, se podría añadir, para la familia que la padece, sostiene y se desespera con ella).

No obstante, hay que plantear que es cierto que el trastorno límite nos acerca a un límite, pero de qué tipo de límite se trata. Dicho de otra manera: cuando aludimos a un trastorno límite, ¿con respecto a qué se orienta ese límite?

Vamos a distinguir a lo largo del trabajo tres aspectos acerca de este límite; por un lado, el límite con el que se encuentra el profesional en el diagnóstico de este tipo de pacientes; por otro, la dimensión límite de su clínica; y, finalmente, la vivencia límite que se infiltra en el paciente.

\section{Sus comienzos}

¿Cómo surge el trastorno límite en la literatura psiquiátrica? La aparición del trastorno límite en la nosología psiquiátrica se produce a causa de una sorpresa clínica o de una dificultad diagnóstica, esto es, a partir de una rareza en la clínica. Son, podríamos decir, los casos raros de la clínica. Por eso, como bien se comprenderá, el trastorno límite siempre ha caminado, en cierta forma, en tierra de nadie.

El trastorno límite, como concepto o idea -aun cuando su interés comience a perfilarse a partir de los años treinta-, surge a mediados de los años cincuenta para 
tipificar un tipo de clínica que no se ajusta al modelo clásico de las neurosis, aunque tampoco al de las grandes psicosis. De esta forma, en la eclosión clínica que comienza ya a perfilarse en la naciente sociedad de consumo, en los años cincuenta viene a surgir un tipo de paciente que no se ajusta a la nosología clásica de neurosis ni psicosis. De ahí una pregunta: ¿son realmente casos nuevos en la clínica, o bien es su demanda en los dispositivos asistenciales la que viene a permitir ahora su descubrimiento y relanzamiento nosológico?

Hay que recordar, en este sentido, que algunos pacientes con los que Freud ya trabajaba en los Estudios sobre la histeria (1) de finales del siglo XIX, podrían ser catalogados, claramente, como trastornos límite. Luego su antigüedad clínica es, a mi modo de ver, anterior a su eclosión y preocupación tardía en el campo de la nosología psiquiátrica. Sin embargo, debe apuntarse una hipótesis: si en la actualidad hay muchos casos que pueden ser contemplados desde esta perspectiva nosológica psiquiátrica -es decir, que de la rareza se ha pasado a una cierta asiduidad en la clínica, siempre y cuando se les sepa encuadrar y escuchar-, es porque hay que pensar que existe algo en esta época de cambios rápidos, caracterizada por su falta de límites, que favorece este tipo de clínica y vivencia límite (2; 3). Porque, a mi modo de ver, la fenomenología clínica es permeable a la época, aun cuando la estructura clínica no lo sea. Y subrayo la expresión «época de cambios rápidos», para transmitir la idea de que hay algo en las transformaciones socio-culturales vertiginosas contemporáneas que no se puede integrar y elaborar suficientemente, ni en el individuo ni en las propias estructuras sociales en las que está insertado, generándose, así, un resto de malestar y errancia. Y es que la falta de límites siempre genera confusión.

En este sentido, cabría formularse una pregunta: ¿existe alguna relación entre la eclosión de este tipo de trastornos límite y la caída o el declive de la función paterna?

Si la neurosis, tal como Freud la formula, alude al marco de una prohibición como garantía de estabilidad psíquica -haciendo de esta prohibición, y el deseo implícito en ella, el baluarte del síntoma neurótico (fobias, obsesiones, conversiones, etc.)- ¿será entonces el declive de la función paterna, en esta sociedad de cambios rápidos, el estandarte de una clínica del vacío y la impulsividad, precisamente por esa difusión de límites que sí garantizaba el padre prohibidor en la neurosis?

¿Cómo irrumpió, entonces, el trastorno límite en la nosología psiquiátrica? El trastorno límite es un concepto que se inserta en el campo de la psiquiatría, a partir del marco del psicoanálisis, precisamente por la importancia que van a ir adquiriendo en su desarrollo nosológico los trastornos de personalidad. Hay que recordar, por otra parte, el gran poder y auge que tuvo el modelo psicoanalítico en el campo de la psiquiatría y sus clasificaciones, durante los años sesenta, en los 
Estados Unidos. De este modo, la llegada de «nuevos» pacientes a los servicios públicos, en el albor de una sociedad de consumo de cambios vertiginosos, introduce un tipo de clínica que no se ajusta exactamente ni al canon de las neurosis, ni al baluarte de las grandes psicosis. Eran así, aparentemente, pacientes de un orden clínico distinto.

En este contexto de errancia diagnóstica comenzaron a plantearse diferentes nombres para todos ellos: esquizofrenia ambulatoria (Zilboorg), personalidad como si (Helene Deutsche), esquizofrenia seudoneurótica (Hoch y Polatin). Más tarde, Kingt, en la discusión de los estados límites; y Frosh, analizando como «carácter psicótico» esta nueva clínica, plantearon que estos pacientes límite estaban al filo de la psicosis, pero no eran propiamente psicóticos. Me parece muy interesante la apreciación que realizan de «al filo de la psicosis» para encuadrar a todos estos pacientes, porque sitúa claramente su problemática y dificultad diagnóstica. Posteriormente, Schmideberg, en 1959, propondrá ya que el trastorno límite resulta un verdadero trastorno del carácter; y todavía, en 1968, autores como Grinker y colaboradores introducirán la descripción de la personalidad límite dentro de un detallado análisis empírico (4). Finalmente, será Otto Kernberg quien ha intentado estructurar los mecanismos defensivos primitivos y las relaciones objetales interiorizadas patológicas de estos pacientes dentro de su esquema de la organización límite de la personalidad, siguiendo el modelo de M. Klein (5).

En síntesis, para algunos de estos autores el trastorno límite acabaría incluyendo un tipo de pacientes que, bajo un ropaje de corte neurótico, hacen de la hostilidad básica (ira inapropiada o manifestaciones de mal genio), la presencia de alteraciones de la identidad (auto-imagen distorsionada, identidad sexual confusa, objetivos a largo plazo indefinidos, errancia en la vida), la sensación de vacío y el aburrimiento constante, así como la inestabilidad afectiva, la conducta impulsiva y agresiva y la transferencia inestable, un nuevo modo de clínica que alude a un marco que no encaja completamente ni en la neurosis ni en la psicosis.

\section{Modos de mirar, modos de escuchar}

Vemos, así, cómo en ciertos ambientes clasificatorios el trastorno límite podría ser concebido como una entidad clínica claramente diferenciada, aunque situada entre la neurosis y la psicosis. Dicho de otra manera: el trastorno límite vendría a ser como un trastorno que, bajo un perfil clínico diferencial, se mostraría independiente entre el modo de funcionar neurótico (conversiones y somatizaciones, angustias, fobias, obsesiones o rituales -que, dicho sea de paso, también configura la supuesta normalidad-) y el modo de funcionamiento psicótico (fenómenos elementales y tramas delirantes). 
Ahora bien ¿define la fenomenología claramente la problemática esencial del sujeto? Dicho de otro modo, ¿nos orientan los síntomas de un modo decisivo acerca de la problemática existente, en el sujeto, hasta el punto de definir su núcleo conflictivo esencial?

A mi modo de ver, la fenomenología no da suficientemente cuenta de la problemática esencial en el sujeto, aun cuando no se la pueda despreciar. Y es que tengo que precisar que los psicoanalistas de orientación lacaniana no estamos demasiado apegados a los síntomas en nuestra clasificación, en tanto que sabemos que éstos no aluden exactamente a la estructura del sujeto, que es lo esencial. Es una cuestión ciertamente doctrinaria y nosológica, pero también de principios prácticos. Porque, en cierto modo, este rigor más específico que se exige en el diagnóstico, y que apunta a la estructura, no es caprichoso, dado que, como bien se comprenderá, no sirve cualquier práctica para cualquier sujeto. De ahí que a la hora de atender a un paciente sea preciso tener muy en cuenta el diagnóstico, más allá de sus síntomas, para poder fundamentar la dirección de la cura. Luego, el diagnóstico, más allá de su fenomenología, es un elemento esencial en la clínica psicoanalítica para orientar su dirección.

En este sentido, también el campo lacaniano se ha hecho eco de esta «nueva» clínica y trata de abordar este tipo de pacientes tan actuales, bajo el modelo de los inclasificables y la psicosis ordinaria, sin obviar completamente el prisma de las neurosis en su dilucidación diagnóstica. Porque, si bien los inclasificables pueden ser entendidos, en ocasiones, como un modo de psicosis no desencadenada, también pueden ser pensados como un impasse en el diagnóstico, que obliga a una escucha expectante. Porque hay dos momentos en la práctica clínica: uno, cuando recibimos al paciente en su singularidad, sin compararlo con nadie, como lo inclasificable por excelencia; es éste un momento en donde pensamos que las clasificaciones son semblantes y lo único que existen son los individuos. Pero hay un segundo momento, diríamos estructuralista, cuando lo referimos a tipos de síntomas y a la existencia de una estructura en el sujeto (6). En esta lógica está en juego un marco estructural de carácter discontinuista y conceptual, en donde el nombre del padre, siguiendo la primera enseñanza de Lacan, establece sus diferencias.

Entonces, ¿los inclasificables de la clínica lacaniana son, en realidad, una nueva estructura que complementa la ya clásica (neurosis, perversión y psicosis), o bien son simplemente un nuevo marco clínico que obliga a replantear todo el modo de pensar la clínica, siguiendo la última enseñanza borromea de Lacan, de carácter elástica y fundada en la generalización del nombre del padre'?

1 «Porque si un delirio puede ser metaforizante, como plantea Lacan, es porque cierta articulación de saber puede funcionar como Nombre del Padre» (7). 
En este sentido también podríamos pensar el trastorno límite como un modo muy particular de anudamiento o funcionamiento de la estructura, bien con respecto a la neurosis, bien con respecto a las psicosis ${ }^{2}$, en donde aquí el límite estaría del lado del profesional en su capacidad y sutileza diagnóstica. Y modo particular de anudamiento cuya vivencia límite y errancia en la vida nos podría marcar y señalar una peculiaridad en la propia estructura. De este modo, el trastorno límite no sería propiamente una estructura distinta, sino simplemente una clínica del síntoma (8) cuyo diagnóstico requeriría una sutileza ínfima del detalle clínico (9). Aquí lo que se pondría en relevancia sería poder acechar y pensar los signos, a veces, ínfimos de la forclusión como mecanismo estructural de la psicosis. Podríamos pensar, por ejemplo, en una excesiva pregnancia de lo imaginario unida a un anclaje simbólico bastante insuficiente, o bien en una relación de extrañamiento entre el yo y el cuerpo (10); pero, también, en una fijación y dialectización imposible con respecto a un entramado conflicto supuestamente edípico, o en el ejercicio desenfrenado y compulsivo de la pulsión, aunque desconectado ya de toda captura en la dialéctica del discurso (11).

Sin embargo, habría también casos en los que podríamos concebir una falta de captura esencial por lo simbólico, que nos transmitiría un sujeto caracterizado por un cierto tipo de flotamiento o inconsistencia esencial. Términos como ausencia de personalidad, debilidad de su inscripción en lo simbólico, extravío de un imaginario sin yo o identificaciones erráticas sin cristalización posible, vendrían a ser como formas de definir un modo de funcionar o de estar en la vida caracterizado por su escasa consistencia o entereza. Y todo ello sin olvidar, en algunos de estos casos denominados como límite, la variabilidad en el funcionamiento de la represión -que no es otra sino más que las peculiaridades variables en el funcionamiento del nombre del padre-, y que igualmente podría crear dificultades en el diagnóstico ${ }^{3}$.

Resuena, aquí y ahora, toda la problemática siempre interesante entre histeria y psicosis, que nos obliga a distinguir entre queja histérica acerca de la vida y sus desgracias o vivencia de muerte del psicótico.

No son éstos, en fin, más que diferentes aspectos que nos indicarían, en los denominados trastornos límites, una falla relevante, aunque a veces muy sutil, en la constitución del sujeto. De ahí que, a mi modo de ver, no sólo se requiera cautela y paciencia clínica en la atención de este tipo de pacientes, sino también un

2 Donde el sujeto neurótico estaría más del lado del amor que del odio, de los síntomas que de los fenómenos, de la represión que de la forclusión.

3 «Pero el padre está en una posición metafórica si y sólo si la madre lo convierte en aquel que con su presencia sanciona la existencia del lugar de la ley. Queda, pues, un inmenso margen para las formas y los medios con los que esto se puede realizar, porque es compatible con diversas configuraciones concretas» (12). 
ORIGINALES Y REVISIONES

exquisito rigor clínico para poder ahondar y definir este tipo de casos raros de la clínica. Y todo ello sumado a la necesidad que tenemos de clarificar previamente la estructura del sujeto que se trata, puesto que de ahí se desprende la propia dirección de la cura.

\section{Un faro de orientación en los trastornos límite}

Por eso es interesante, al hilo de estos casos raros de la clínica, que nos podamos plantear una pregunta que nos permita orientar nuevamente el caso. Podríamos pensar entonces, y es una hipótesis a desarrollar, qué se privilegia en el encuentro con estos sujetos: ¿Falta en ser o falla forclusiva? ¿Vacío o agujero? ¿Misterio o enigma? ¿Duda o certeza? Veamos.

Y es que la palabra límite también alude a una cuestión vital que se aprecia bien en la clínica con estos sujetos y que presupone una cierta dificultad, a corto plazo, en el manejo y pronóstico del caso. Porque son conocidas la falta de límites y la impulsividad con que estos sujetos se mueven alrededor de un vacío o un agujero que les angustia, y que o bien tiene que ver con una «pregunta» que no está siendo suficientemente formulada, y por tanto aclarada, o bien tiene que ver con el hecho de que «la pregunta viene de allí donde no hay significante» (13), en alusión a la ausencia del significante del Nombre del padre.

Precisamente, el tratamiento psicoanalítico parte de la premisa de que en el síntoma opaco o en la errancia del sujeto en la vida, junto con su angustia como encuentro con lo imposible, está implícita una «pregunta» que el tratamiento psicoanalítico debe tratar de formular, acotar y constituir en el contexto de las entrevistas preliminares.

Porque, para orientarnos en el diagnóstico, también podríamos entender toda esta temática acerca del límite como una vivencia del sujeto con relación a una «pregunta» que se instala en él en un momento de su encrucijada vital, o, todo lo contrario, que la pregunta simplemente no ha existido, como tal, nunca en él (14). $\mathrm{Y}$ es que como dice Lacan: «Estamos seguros que los neuróticos se hicieron una pregunta. Los psicóticos, no es tan seguro. Quizá la respuesta les llegó antes que la pregunta; es una hipótesis. O bien la pregunta se formuló por sí sola, lo cual es impensable» (15).

Precisamente es esta «pregunta», cuya constitución y dilucidación forma parte del tratamiento psicoanalítico, la que es preciso hacer resonar en el contexto de las entrevistas preliminares para poder afrontar la cura. Pregunta vital al hilo de la propia existencia, cuya respuesta tendría que venir de la mano de todo ese andamiaje de posibles respuestas, siempre inconclusas, que el sujeto ha ido labrando a lo largo de su travesía edípica. Y es que esta «pregunta» que exige una 
respuesta, y que el tratamiento psicoanalítico debe hacer resonar, en su formulación, en las entrevistas preliminares y en la cura -mediante la construcción fantasmática-, es lo que nosotros concebimos como uno de los elementos fundamentales en el desencadenamiento del cuadro tanto por su respuesta insuficiente o inconclusa, como, también, «porque la pregunta viene de allí donde no hay significante» (16).

Debe recordarse ahora en qué consiste la travesía edípica para tratar de interrogar esta «pregunta» ${ }^{4}$ que el análisis debe hace resonar en el contexto de la cura, siempre y cuando en verdad se haya podido formular previamente.

El Edipo es el modo como un sujeto constituye su identidad yoica ${ }^{5}$, su identidad sexual y su elección de objeto sexual, pero también su modo particular de goce. Dicho de otra manera: el sujeto a lo largo de la travesía edípica va dilucidando y configurando ese fantasma -su forma de ser- que encaminará su futura posición en la vida, la sociedad y la cultura, a la vez que le permite ir estableciendo ese marco necesario de separación del entorno familiar. De este modo, construcción del fantasma, como modo de respuesta al enigmático deseo del Otro, y separación del entorno familiar son elementos fundamentales para poder disfrutar de la vida e insertarse en el ámbito social como sujeto deseante. Es interesante todo este asunto, porque es esto mismo lo que percibimos como alterado y confuso en los denominados trastornos límite. Y es que la dilucidación vital, en el contexto del Edipo, siempre arrastra una cierta sensación de identidad y vitalidad en el sujeto, en tanto que esta cristalización no es más que el entramado imaginario y simbólico que define su modo de ser, estar y funcionar en la vida.

Es muy compleja toda esta cuestión enigmática que representa el encuentro con la vida. Y resulta un enigma cuya respuesta siempre encubre, en realidad, un agujero que a mí, personalmente, me invita a recordar ahora esa forclusión generalizada a la que alude Lacan en su última enseñanza, un agujero que podríamos plantear con relación al propio enigma de la vida.

Por eso, los clínicos han descrito gran variedad de síntomas a la hora de abordar este tipo de sujetos límite en su confrontación con lo real que aluden, precisamente, a toda esta problemática en la constitución del sujeto (identidad yoica, identidad sexual y elecciones objetales) a lo largo de su travesía edípica. De esta forma términos como difusión de identidad (vacío crónico, autopercepciones contradictorias, conductas paradójicas), ansiedad crónica difusa, libre y flotante, neurosis polisintomáticas, tendencias sexuales perversas polimorfas o neurosis y adic-

\footnotetext{
4 Pregunta vital que en la neurosis viene marcada por «soy hombre o mujer», en la histeria, «estoy vivo o muerto», en el obsesivo.

5 Precisamente en el argot clínico el término difusión de identidad alude a la confusión yoica que padecen estos sujetos límite.
} 
ORIGINALES Y REVISIONES

ciones por impulso; pero también las personalidades prepsicóticas clásicas (paranoide, esquizoide, hipomaníaca) o los trastornos del carácter de menor nivel (personalidades infantiles y narcisistas típicas, personalidades como si, personalidad antisocial) (17), reflejan modos demasiado extensos e inespecíficos a la hora de definir este tipo de clínica. Sin embargo, este cóctel sintomático, difuso e inestable que embarga a toda esta nosología, debería responder a alguna cuestión mucho más específica que la pura sintomatología. Es decir: no se trataría simplemente de puros fenómenos que acontecen en un sujeto, sino más bien de una particularidad que afecta a la propia estructura en juego.

Por eso no es azaroso que este tipo de clínica que acompaña al denominado trastorno límite, debute, en ocasiones, en un momento muy determinado, preferentemente en la adolescencia, precisamente cuando el sujeto se ve llamado a responder ante las propias exigencias o preguntas de la vida (el encuentro con la sexualidad y con el mundo social) mediante toda esa configuración fantasmática (identidad yoica y sexual, elecciones de objeto), siempre insuficiente, que ha sido labrada en el contexto de la estructura edípica. Y ahí, en ese momento de confrontación con la vida, algo puede resistirse y mostrarse opaco para el sujeto, dejando al descubierto un vacío -o una falta- que siempre había estado velado, o bien irrumpiendo un agujero que siempre existió. Tendríamos, así, dos modalidades distintas: la crisis o conmoción fantasmática, y la caída de lo simbólico y la succión por el agujero que precipitarán el malestar particular y la eclosión de la clínica que configurará el cuadro nosológico.

Ahondando ahora aún más en toda esta cuestión acerca del límite y la «pregunta» que acontece en el sujeto -y que el tratamiento psicoanalítico trata de hacer resurgir ante lo inexplicable del síntoma-, podríamos decir que al sujeto se le plantea en la encrucijada de su vida una «pregunta» y se le exige una respuesta. Si el sujeto afronta esta cuestión desde el lado del misterio, podrá entonces sorprenderse mediante el encadenamiento o la sustitución de las palabras (metonimia y metáfora), para encontrar así, en su búsqueda particular, un punto de sentido que a modo de respuesta pacifique su malestar con relación a una falta en ser que le aqueja y le interroga, y que define su propia vivencia límite. Pero, para ello, es preciso que el sujeto disponga de un instrumento que le oriente en ese misterio que le interroga acerca de la falta en ser, en ese momento de su vida. Precisamente, en psicoanálisis se conoce como función fálica -función del nombre del padre- a ese instrumento que permite velar y orientar, en cierto modo, la falta. Sin embargo, si el sujeto afronta esta pregunta desde el lado del enigma, entonces no hay respuesta posible, «porque el enigma precisamente pone a prueba la relación del significante con el significado. Es incluso la evidencia de su no-relación» (18). Y aquí, en este segundo caso, lo que aparece más bien es una falla forclusiva, un agujero, que deja en evidencia no ya una falta en ser que garantiza el deseo mediante todos 
esos objetos sustitutos que remedan lo perdido, sino más bien un desierto de significación, una sensación de perplejidad con relación a la «pregunta», que, a veces, es preciso captar en el sujeto mediante signos ínfimos.

Tenemos, así, dos diferentes modos de vivenciar el límite ${ }^{6}$, que aluden ciertamente a estructuras bien distintas. Por un lado, la relación entre una «pregunta» que produce una conmoción fantasmática que quedará integrada en lo simbólico y que precipita en el sujeto una crisis; hay aquí continuidad y no ruptura en la experiencia, por más descalabro fantasmático que se vivencie o se perciba desde el exterior. Y, por el otro, una «pregunta» que, en tanto, agujereada, produce en el sujeto ruptura, caída de lo simbólico y succión por el agujero. Y es que, en este segundo caso, en tanto que la «pregunta» viene de allí donde no hay significante, es más bien el encuentro con un agujero el que precipitará en el sujeto esa sensación de perplejidad que será vivida como certeza.

Vemos así, por una parte, vacío, misterio y «pregunta» del lado de lo simbólico, que precipitarán en el sujeto neurótico una crisis, precisamente por la conmoción que acontece en ese mundo fantasmático que vela lo real. Y, por otra parte, agujero, enigma y «pregunta» desde el lado de lo real, que precipitan un desencadenamiento psicótico que puede ser, sin embargo, muy puntual. Son ciertamente dos acontecimientos diferentes, pero también dos modos de encuentro con la vivencia límite o lo real bien distintos. Y es que mientras que, en el primer caso, se evidencia la presencia de un instrumento, el falo, que puede instrumentalizar el encuentro con lo real, en el segundo caso la ausencia del falo deja al sujeto completamente inerme y desamparado en ese encuentro con lo imposible de decir.

Sin embargo, no siempre las cosas se presentan, exactamente, bajo el prisma de la crisis o la ruptura que anuncia esa vivencia límite en ese encuentro con lo real. En ocasiones, no se percibe en el sujeto ninguna crisis o ruptura ostensible; tan solo una vivencia límite difusa -sensación de vacuidad-, que alude a una falla o a una inconsistencia esencial de la propia estructura. Son esos casos en los que tan solo apreciamos una falta de sostén y precariedad, que nosotros podemos interpretar, en realidad, como una falla forclusiva en la propia estructura. Verdaderamente, aquí la pregunta, como tal, permanece informulable. Y pienso que, en estos casos, el capítulo de la psicosis ordinaria, en la órbita lacaniana, nos puede orientar (20).

Ahora bien: a la hora de abordar este vacío o agujero, en función del caso, creo que habría que concebir lo que podríamos definir como una cierta articulación entre la angustia y esa respuesta, tan frecuente, en este tipo de pacientes: la conducta impulsiva. Por un lado, tendríamos la impulsividad y la búsqueda de un objeto que calme la angustia, siguiendo el correlato de un fantasma que vela el

6 Entendiendo aquí el límite, como el encuentro con lo real o lo imposible de decir (19). 
vacío. Por el otro, la dimensión de un agujero, que en tanto muestra abruptamente lo real, se hace entonces necesario calmar rápidamente y sin demora. Quizás la sutileza de la clínica y su escucha, más allá de la comprensión del propio impulso, nos podría orientar también en las diferencias entre estos dos modos de atemperar el vacío o el agujero mediante la impulsividad.

Tenemos así, de nuevo, dos tipos de pacientes, que, aun cuando se puedan mostrar parecidos en su fenomenología, son, sin embargo, bien distintos. Y es que, mientras en unos domina el vacío, efecto de la falta en ser, en otros predomina el agujero en la significación fálica como una falla esencial en la estructura. Son las diferencias entre el impasse edípico del sujeto neurótico en su encuentro con lo imposible y el propio agujero edípico del sujeto psicótico. Términos como fragilidad yoica ${ }^{7}$ o conmoción fantasmática del lado de los sujetos que están encuadrados en el perfil neurótico, frente a esta inconsistencia o falla esencial en la estructura del lado de los sujetos psicóticos, nos anuncian también dos modos de relación con el Lenguaje y la palabra bien distintos. Y ambos nos revelarían dos tipos de pacientes, que, aun cuando puedan manifestar ocasionalmente fenómenos aparentemente similares, reflejan, sin embargo, problemáticas y abordajes diferentes.

Finalmente, quisiera abordar otro estatuto acerca del límite, al hilo de la experiencia analítica como abordaje de lo real. Porque, en sintonía con todas estas cuestiones que nos interrogan acerca de este modo de vivenciar el límite o el encuentro con lo real -mediante el vacío-misterio del neurótico o el agujeroenigma del psicótico-, podríamos plantear que, si bien la pregunta por la vida es siempre un enigma esencial insertado en el corazón del ser parlante, éste puede, sin embargo, quedar recubierto bajo el amparo de unas palabras que hacen del propio enigma un simple misterio por desvelar. Y, mientras que el misterio es aquello que es abordable mediante el encadenamiento y la sustitución de las palabras, el enigma sin embargo, siempre se muestra resistente y opaco a las mismas. De cada uno, en función de su relación con la verdad y la castración, dependerá insistir en el enigma, entendiéndolo aquí como misterio, o bien introducir en él la función del silencio, esto es, el cese de las palabras como un modo de aceptar todo aquello que las palabras no pueden cernir.

De ahí que, mientras que la pregunta en juego, en la neurosis y su síntoma, sea siempre un misterio que invita a hablar en esa búsqueda de resolución del mismo, el propio curso de las palabras debe concluir, en la dirección de la cura, en un enigma que promueva e invite al silencio. Y es que, en la neurosis, enigma quiere decir ahora que, en el lugar de la significación, lo que aparece es, nuevamen-

7 ¿Cómo distinguir, entonces, la identificación frágil de la histeria, que sí está mediatizada por la función fálica, de esa falla forclusiva en la psicosis que carece sin embargo, de esta significación? 
te, un agujero. Pero es ya, sin embargo, un agujero vaciado que no precipita perplejidad, como en el sujeto psicótico, sino simplemente silencio. Por eso mientras que, en el inicio de la cura, el misterio del síntoma en la neurosis está más en relación con un vacío que se trataría de rellenar mediante el sentido, el agujero que finalmente se instala en la cura estaría más bien del lado de la no-significación, porque en verdad, «no hay» significante ${ }^{8}$. De ahí que aquello que inicialmente diferencia al neurótico del psicótico en esa vivencia límite que supone el trauma con lo real acaba finalmente por asemejarles -vacío agujereado y agujero-, aunque de un modo bien distinto. Si en la neurosis el encuentro con el misterio del síntoma invita a hablar hasta llegar a agotar las palabras en su propio sentido -que no es más que el intento de cernir el sin sentido que palpita entre ellas-, el enigma que finalmente brota entre las propias palabras invita a un silencio, pero también a lo que podríamos denominar una certidumbre acerca de lo posible. Y es que, mientras que el misterio tiene palabras que buscan aportar incesantemente un sentido -que siempre esconde el sinsentido- a lo misterioso del síntoma, el enigma sólo tiene como respuesta la locura y la certeza $^{9}$, o bien el silencio y la certidumbre acerca de posible, pues, como diría Wittgenstein: «De lo que no se puede hablar mejor es callarse».

Por último, y en el contexto de la cura con estos pacientes, tanto si hay crisis como caída de lo simbólico y succión por el agujero (ya diferenciando de este modo dos formas diversas de vivenciar el límite, que aluden ciertamente a estructuras distintas), es preciso una orientación y acompañamiento que introduzca un cierto apaciguamiento en este encontronazo con lo real, a la vez que un tipo de invención que permita a estos sujetos «límite» apañárselas con este imposible de decir. En esto consistiría el tratamiento con este tipo de pacientes. Tratamiento largo, sin duda, y no exento de complicaciones e impasses. Pero, sólo de este modo, el sujeto podrá afrontar el encuentro con la vida, así como la separación ineludible con la familia que se precisa para encauzar su existencia en función del deseo.

\footnotetext{
8 Lacan aludirá a expresiones como «no hay relación sexual», o bien, «falta el significante La mujer» para dar cuenta de toda esta temática (21).

9 La religión es, en este sentido, un tipo de certeza «loca» que, sin embargo, hace lazo social, en tanto que promueve frente al enigma de la vida un tipo de creencia que impone certidumbre.
} 


\section{BIBLIOGRAFÍA}

(1) Freud, S., Estudios sobre la histeria, Madrid, Biblioteca Nueva, 1973.

(2) López Herrero, L. S., «Histeria y Psicoanálisis: una pareja en apuros», Cuadernos de Psicoanálisis de Castilla y León, 2001, 3, pp. 23-38.

(3) LóPEz Herrero, L. S., «Somatizaciones: cuando el cuerpo goza», VI Jornadas Asociación Castellano-Leonesa de Salud Mental, 2000, pp. 181-189.

(4) Kaplan, H. I.; Sadock, B. J., Tratado de Psiquiatría, Barcelona, Ediciones Científicas y Técnicas, 1992, p. 970.

(5) Kernberg, O. F., Trastornos graves de la personalidad, México, Manual Moderno, 1987.

(6) VV. AA., Los inclasificables de la clínica psicoanalítica, Buenos Aires, Paidós, 1999, pp. 402-404.

(7) Ibídem, p. 412.

(8) Ibídem, p. 201.

(9) Ibídem, p. 202.

(10) Ibídem.

(11) Ibídem.

(12) LaCan, J., Seminario 5, Buenos Aires, Paidós, 1999, p. 202.

(13) LaCAN, J., Seminario 3, Buenos Aires, Paidós, 1992, p. 289.

(14) Ibídem, p. 288.

(15) Ibídem.

(16) Ibídem.

(17) Kernberg, O. F., Trastornos graves de la personalidad, México, Manual Moderno, 1987, pp. 8-9.

(18) VV. AA., Los inclasificables de la clínica psicoanalítica, Buenos Aires, Paidós, 1999, p. 21.

(19) LaCAn, J., Seminario XVII, Buenos Aires, Paidós, 1992, p.131.

(20) Miller, J. A., y otros, La psicosis ordinaria, Buenos Aires, Paidós, 2004.

(21) LaCan, J., Seminario XX, Buenos Aires, Paidós, 1995, pp 87-89.

* Luis-Salvador López Herrero, Médico especialista Medicina Familiar y Comunitaria. Psicoanalista. Tutor de la Unidad Docente de Medicina Familiar y Comunitaria de León. Centro de Salud José Aguado. León; María Elena González Lucas, Residente 3..$^{\circ}$ Medicina Familiar y Comunitaria de León; Laura Linares Álvarez, Residente $2^{\circ}$. Medicina Familiar y Comunitaria de León. Correspondencia: Luis-Salvador López Herrero, C/ Páramo, n. ${ }^{\circ}$ 1, 5. ${ }^{a}$ A. Esc. 3. León. 24006. luis_salvador@terra.es

** Fecha de recepción: 13-IV-2007 (aceptado el 07-IX-2007). 